\title{
Mistletoe Extract Viscum Fraxini-2 for Treatment of Advanced Hepatocellular Carcinoma: A Case Series
}

\author{
Richard T. Lee ${ }^{a} \quad$ Peiying Yang ${ }^{b} \quad$ Asrar Alahmadi $^{a} \quad$ Jennifer McQuade ${ }^{c}$ \\ Eric Yuan $^{a}$ Analisa Difeo ${ }^{d}$ Goutham Narla $^{e}$ Ahmed Kaseb $^{f}$ \\ aDepartment of Medicine, University Hospitals Cleveland Medical Center \& Case Western \\ Reserve University, Cleveland, OH, USA; 'bepartment of Palliative, Rehabilitation, and \\ Integrative Medicine, The University of Texas MD Anderson Cancer Center, Houston, TX, \\ USA; 'Department of Melanoma Medical Oncology, The University of Texas MD Anderson \\ Cancer Center, Houston, TX, USA; d Departments of Obstetrics and Gynecology, University

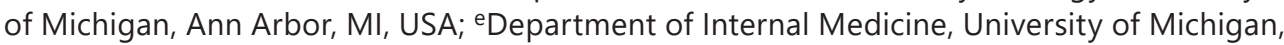 \\ Ann Arbor, MI, USA; f Department of Gastrointestinal Medical Oncology, The University of \\ Texas MD Anderson Cancer Center, Houston, TX, USA
}

\section{Keywords}

Fraxini $\cdot$ Hepatocellular carcinoma $\cdot$ Mistletoe lectin

\begin{abstract}
Background: Hepatocellular carcinoma (HCC) is the fourth leading cause of death from cancer worldwide, and for advanced HCC the prognosis is poor. Preliminary studies indicate mistletoe extracts may have anticancer activity for HCC. Methods: A prospective observational case series of advanced HCC patients that chose to take a mistletoe extract called viscum fraxini-2 (VF-2) alone for treatment. Time on treatment, imaging, and laboratory values were collected for descriptive analyses. Results: A total of 12 patients with advanced HCC enrolled onto the protocol, and 10 patients had data available for evaluation. The majority were male $(10 / 12)$ with a median age of 64 (SD 11). Most patients had received sorafenib therapy (9/12) and had varying Child-Pugh classes (A-4, B-6, C-2). Treatment with VF-2 ranged from 1 to 36 weeks with a mean of 12.3 weeks (SD 12). Six patients received 8 weeks of treatment, and 3 patients received 12 or more weeks of treatment. For patients that received at least 4 weeks of treatment, the average AFP value stabilized during the first 4 weeks of treatment. Two patients experienced an AFP decrease of $>30 \%$, approximately 37 and $40 \%$ decreases at the nadir. One patient had stable disease of 9 months. Major side effects were fever, fatigue, rash, and local injection site reaction of swelling, redness, and tenderness. Conclusion: This case
\end{abstract}


series of advanced HCC indicates that mistletoe extract VF-2 may have potential biological activity against HCC for selected patients. Research is needed to identify the active compound and predictive markers of response.

\section{Introduction}

Liver cancer remains a prevalent cause of cancer worldwide, the fourth leading cause of death from cancer [1]. Hepatocellular carcinoma (HCC) is the most common liver cancer and accounts for the vast majority of cases. Although the incidence of HCC is on decline in certain countries in Asia with increased vaccination for hepatitis B, it has been increasing in other counties including the United States [2]. Less developed countries have age-standardized rates approximately double of developed countries with men slightly higher than women: men 17.8 versus 8.6 and women 17.0 versus 7.1 , respectively. The main risk factors remain hepatitis B virus (HBV) and hepatitis $\mathrm{C}$ virus (HCV) infections leading to cirrhosis. Alcohol, non-alcoholic fatty liver disease, obesity, toxins (ex. aflatoxin and vinyl chloride), and smoking are also important risk factors with variable rates in different regions of the world [3]. Vaccination efforts for HBV along with improved hygiene and sanitation have led to dramatic decreases in cases in Taiwan, Japan, and China. Recent therapies for the treatment of HCV have shown high success rates, but for patients who already have advanced cirrhosis, the risk of developing HCC remains elevated [4]. However, the incidence in other countries appears to be rising, including in some developed countries such as the United States where rates have tripled from 2.6 per 100,000 in 1975 to 8.6 per 100,000 in 2011. Projections indicate a continued rise in HCC in the coming decade [5].

In the United States, overall 5-year survival for all stages is less than 15\%. In general, less than $20 \%$ of patients diagnosed with HCC are good candidates for surgical resection based on stage of disease and comorbidities as a curative therapy, with higher cure rates in Asia. The small percentage of patients that have limited disease as defined by different criteria (e.g., Milan criteria) and liver cirrhosis may still be eligible for liver transplantation as a curative approach, with 5-year success rates as high as 70-80\% [6]. Tumor ablation using methods including radiofrequency ablation (RFA) remains a potential curative options for those not able to undergo surgery or liver transplantation, and with limited size tumor $(<3 \mathrm{~cm})$. However, intrahepatic recurrence is common in these cases, with a 5-year recurrence rate up to $75 \%$ and overall survival of $50-60 \%$.

Patients with advanced HCC that is not amenable to curative therapies can have their cancer managed by a variety of local regional therapies such as transarterial chemoembolization (TACE), transarterial radioembolization, and external beam radiotherapy (EBRT). For patients with progressive disease, sorafenib and lenvatinib are approved for first line therapy and have demonstrated a limited survival benefit of an additional 2-3 months [7]. Immunotherapies (nivolumab and pembrolizumab) have been approved for advanced HCC based on phase II studies; however, recent phase III studies did demonstrate an overall survival benefit. Cabozantinib and ramucirumab have been approved for second-line therapy, but also have a limited benefit of approximately 2 months. Most recently, atezolizumab and bevacizumab have been approved in the United States for first-line treatment with significant improvements survival [8]. Despite these advances, these therapies have a limited response rate of $<30 \%$ with over $90 \%$ of patients eventually failing treatment. Thus, given the limited benefit of systemic treatments available for patients with advanced HCC, new targets and therapies are urgently needed.

\section{Karger'}


Patients are increasingly turning to complementary and alternative medicines as a way to treat their cancers. A national survey of adult Americans found that approximately 1 in 6 adults (17.7\%) have used herbs and supplements (HS) in the past 12 months, and studies show cancer patients' use of HS exceeds $50 \%$, often concurrent with conventional cancer therapy. It is imperative that both patients and oncologists understand the risks and benefits related to the use of these products.

Mistletoe is a parasitic plant that grows on a variety of different trees (ex. pine, ash, apple, oak) and has been used for a variety of ailments as early as the 1500's by Paracelsus. More recently, several randomized clinical trials have been conducted with some demonstrating reduction in symptoms and improved quality of life. Although some cohort and retrospective studies have indicated possible anticancer activity, studies from prospective clinical trials have been more mixed. With regard to HCC, investigators in Egypt have conducted a prospective non-randomized clinical trial of mistletoe therapy (viscum fraxini-2, VF-2) for advanced patients not eligible to pursue other treatments [9]. They demonstrated a combined partial response (PR)/complete response (CR) rate of $20 \%$ and stable disease (SD) rate of $33 \%$ in this study of 120 patients. Based on this promising data, we decided collect clinical data on those patients that decided to pursue mistletoe treatment.

\section{Methods}

An observational protocol was created and IRB approved to allow collection of clinical data for patients with cancer choosing to pursue complementary and alternative therapies (Protocol \#DR-0148). Patients with advanced HCC that had exhausted all other conventional FDA-approved therapies as well as clinical trials (including phase I studies) were informed about the results from the published phase II study in Egypt. Because VF-2 is not FDA approved and not available in the United States, patients obtained the medication directly through the company in Germany (Abnoba, GmbH). Patients were instructed to take VF-2 similar to the phase II study. Patients started at a half dose $(20 \mathrm{mg})$ by subcutaneous injection once per week for 2 weeks after which time they had a follow-up clinical evaluation. If patients tolerated the therapy, they were instructed to increase to full dose $(40 \mathrm{mg}$ ) subcutaneously once weekly. Because of the potential local injection site reactions, patients were instructed to split the injection into two separate sites. Laboratory evaluations (CBC, metabolic panel, liver function, AFP) were drawn at baseline, after 2 weeks, and after 8 weeks of therapy. Imaging with CT or MRI was conducted at baseline and after 8 weeks of therapy. Patients were assessed both by clinical symptoms, laboratory results, and imaging findings. Imaging that showed both signs of tumor growth and tumor regression were deemed to have a mixed response. Patients continued therapy if either PR ( $>30 \%$ reduction in tumor size) or SD were noted and discontinued treatment for progressive disease (PD, $>20 \%$ tumor growth) or toxicity.

\section{Results}

A total of 12 patients with advanced HCC on VF-2 treatment were enrolled on the observational protocol from January 2013 to December 2015. Only 10 patients had data available for evaluation. Patients were mostly male (10 of 12) with a median age of 64 (SD 11) and an Eastern Cooperative Oncology Group (ECOG) performance status of 1-2. Major risk factors for HCC were HCV [5] or alcohol [2] related cirrhosis. All but 1 patient had undergone previous treatments including sorafenib [9], TACE [5], EBRT [5], chemotherapy [3], RFA [1], and

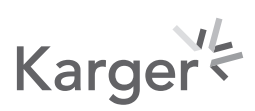




\section{Case Reports in Oncology}

Table 1. Patient demographics

\begin{tabular}{l|l}
\hline Case Rep Oncol 2021;14:224-231 \\
\hline DOI: 10.1159/000511566 & $\begin{array}{l}\text { @ 2021 The Author(s). Published by S. Karger AG, Basel } \\
\text { www.karger.com/cro }\end{array}$ \\
\hline
\end{tabular}

ee et al.: Case Series: Mistletoe for Treatment of HCC

\begin{tabular}{|c|c|}
\hline Age & 62.8 \\
\hline \multicolumn{2}{|l|}{ Gender } \\
\hline Male & 10 \\
\hline Female & 2 \\
\hline \multicolumn{2}{|l|}{ Risk factors } \\
\hline Hepatitis C & 5 \\
\hline Alcohol & 2 \\
\hline Non-alcoholic fatty liver disease & 1 \\
\hline \multicolumn{2}{|l|}{ ECOG } \\
\hline 1 & 6 \\
\hline 2 & 6 \\
\hline \multicolumn{2}{|l|}{ Previous treatment } \\
\hline Sorafenib & 9 \\
\hline TACE & 5 \\
\hline EBRT & 5 \\
\hline Systemic chemotherapy & 3 \\
\hline RFA & 1 \\
\hline $\mathrm{AFP}, \mathrm{ng} / \mathrm{mL}$ & $38,565 \pm 10,3687$ \\
\hline Total bilirubin, $\mathrm{mg} / \mathrm{dL}$ & $2.7 \pm 2.6$ \\
\hline Albumin, g/dL & $3.3 \pm 0.58$ \\
\hline Prothrombin time, s & $15.0 \pm 1.3$ \\
\hline \multicolumn{2}{|l|}{ Child-Pugh } \\
\hline A & 4 \\
\hline $\mathrm{B}$ & 6 \\
\hline $\mathrm{C}$ & 2 \\
\hline $\begin{array}{l}\text { ECOG, Eastern Cooperative Onc } \\
\text { chemoembolization, EBRT, external } \\
\text { quency ablation. }\end{array}$ & $\begin{array}{l}\text { p; TACE, transar } \\
\text { therapy, RFA, rad }\end{array}$ \\
\hline
\end{tabular}

Table 2. Response to viscum fraxini-2 treatment

\begin{tabular}{|c|c|c|c|c|c|c|}
\hline ID & Previous treatment & ECOG & $\begin{array}{l}\text { Child- } \\
\text { Pugh }\end{array}$ & $\begin{array}{l}\text { Metastatic } \\
\text { disease }\end{array}$ & $\begin{array}{l}\text { Weeks on } \\
\text { treatment }\end{array}$ & First assessment \\
\hline 1 & TACE, RT, sorafenib & 1 & $8-\mathrm{B}$ & None & 30 & SD \\
\hline 2 & RT, sorafenib, TACE, HAI & 1 & $5-\mathrm{A}$ & Bone & 7 & $\mathrm{PD}$ \\
\hline 3 & None & 2 & $8-\mathrm{B}$ & None & 4 & Clinical progression \\
\hline 4 & RT, sorafenib & 1 & $7-B$ & Lungs & 17 & Mixed response \\
\hline 5 & TACE, sorafenib & 2 & $5-\mathrm{A}$ & Bone & 5 & $\mathrm{PD}$ \\
\hline 6 & Gemcitabine + bevacizumab, sorafenib & 2 & $7-B$ & None & 2.6 & Clinical progression \\
\hline 7 & Sorafenib, RT, bevacizumab & 2 & $10-\mathrm{C}$ & None & 9 & $\mathrm{PD}$ \\
\hline 8 & TACE, sorafenib & 2 & $10-\mathrm{C}$ & Peritoneal & 1.4 & Clinical progression \\
\hline 9 & TACE, RT, sorafenib, sirolimus + vorinostat & 1 & $5-\mathrm{A}$ & Lungs & 36.4 & SD \\
\hline 10 & Sorafenib & 2 & $5-\mathrm{A}$ & None & 10.3 & PD \\
\hline
\end{tabular}

TACE, transarterial chemoembolization; RT, radiotherapy; HAI, hepatic artery infusion.

hepatic arterial infusion [1]. Patients had a range of Child-Pugh classifications: $A=4, B=6$, $\mathrm{C}=2$. The group included 5 patients with distant metastatic disease (Tables 1,2).

Treatment length ranged from 1 to 36 weeks with an average of 12.3 (SD 12) weeks and a median of 8 weeks. Eight patients were able to receive 4 weeks of treatment and 6 patient received 8 weeks of treatment. Only 3 patients continued therapy beyond 12 weeks of treatment. Best responses were recorded as SD. No CR or PR were observed by imaging. One 


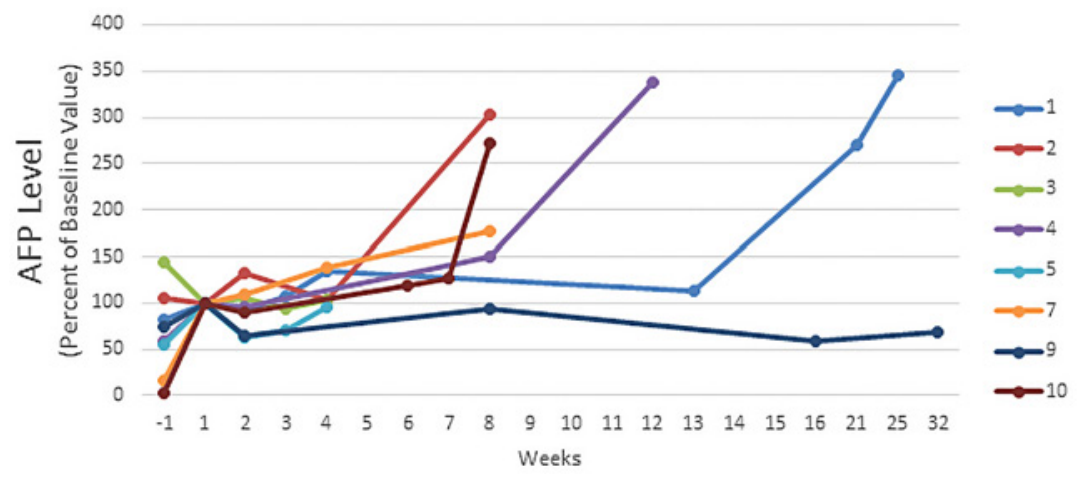

Fig. 1. Changes in AFP during treatment with viscum fraxini-2. AFP, alpha-feto protein. -1 time point indicates AFP level prior to starting treatment.

patient was found to have a mixed response and remained on treatment 17 weeks. A second patient stayed on treatment for 30 weeks until imaging showed PD. The third patient with SD stayed on treatment for 36 weeks and stopped therapy in order to begin treatment on a new phase I clinical trial. This patient later developed brain metastases approximately 1 month after stopping VF-2 treatment (Fig. 1). While most patients had a rising AFP prior to starting treatment with VF-2, on average AFP appeared to stabilize within the first 3-4 weeks among patients who received at least 4 weeks of treatment. Two patients demonstrated a $>30 \%$ reduction of AFP from baseline ( 37 and 40\%), and for 1 patient this remained decreased for 9 months.

Major side effects experienced by patients were fever, fatigue, skin rash, and local injection site reactions (swelling, redness, and tenderness). These side effects slowly resolved in 3-5 days after injection. In 2 cases, patients were hospitalized after beginning treatment due to toxicity. The first patient experienced a severe full body rash and was able to resume therapy after a 1-week pause. The second patient was admitted with worsening hyperbilirubinemia after the first dose and stopped therapy.

\section{Discussion}

This case series examining the efficacy of a mistletoe extract (ME) for the treatment of advanced HCC documents a potential clinical activity of this therapy which was overall tolerable in this group of advanced HCC stage and cirrhosis grade patients. Notably, most patients demonstrated stabilization of their AFP values relative to baseline values. This is relevant in light of recent evidence of correlation between AFP levels and tumor biology. In comparison to the previously published phase II study of VF-2 treatment, almost all of these patients had progressed on systemic therapy, mostly sorafenib. Three patients were able to stay on treatment for 12 weeks, including one that had SD for approximately 9 months. This patient showed SD on imaging and only progressed after stopping therapy in order to enroll in a phase I clinical trial. This data seems to support early clinical studies of MEs for the treatment of advanced HCC.

Studies from prospective clinical trials of MEs have shown clinical activity including potentially improved survival. Among these seven clinical trials of a mixed ME, six different mistletoe preparations were used, making comparisons difficult as each preparation contains 
different amounts of mistletoe lectin (MLs). Early phase clinical trials have been reported with the purified recombinant mistletoe lectin I (MLI), aviscumine, in a variety of advanced cancer types [10]. SD was reported in all three clinical trials including 35\% in a phase II study of stage IV melanoma treated with aviscumine. The most relevant clinical data to this case series comes from a prospective non-randomized clinical trial of a specific ME named VF-2 for advanced HCC [9], which reported an objective response rate of $20 \%$ (including 2 CRs) and SD rate of $33 \%$ among 120 patients.

Both in vitro and in vivo preclinical studies have indicated that ME have anticancer effects in a variety of cells line and animal models, and induce apoptotic or necrotic cell death in a variety of human tumor cell lines in vitro [11]. Among the bioactive components of ME that have anti-cancer activity, MLs are shown to be most potent. ML refers to a heteroprotein complex comprised of an A- and B-chain connected by a disulfide bond. The A-chain has been found to inhibit ribosomes similar in action to ricin, while the B-chain binds to cell surface glycoproteins triggering the internalization of ML. Three MLs (MLI, II, III) have been identified, and the ME viscum fraxini (Fraxini) has the highest concentration of ML, mainly MLI $(>10,000 \mathrm{ng} / \mathrm{mL})$. Numerous in vitro and in vivo studies have demonstrated that ML exerts strong anti-cancer activity in various cancers, including in HCC. Korean ML inhibits the proliferation of both SK-Hep-1 and Hep3B cells, and augment the anti-cancer activity of paclitaxel in Sk-Hep-1 cells. Similarly, the recombinant MLI aviscumine (Melema Pharma), expressed in Escherichia coli, is as cytotoxic as plant-derived ML by induction of apoptosis. More recently, our group has identified downregulation of the c-Myc protein as a potential molecular mechanism for ML-induced apoptosis [12]. This is important given that MYC gene amplification is commonly found in HCC [13]. In mice with inducible MYC gene expression, inactivation of the protein leads to regression of liver tumors, further supporting the dependency of HCC on MYC signaling. Additionally, studies demonstrate that c-Myc amplification is correlated with advanced disease and worse overall survival in HCC [12].

Notably, natural products have been an important source for drug discovery. A review of FDA-approved anticancer therapies found that $41 \%$ of anticancer drugs approved between 1940 and 2010 were either natural or naturally derived. Commonly used chemotherapies originally derived from natural plants include paclitaxel, irinotecan, vincristine, and etoposide. More recently, trabectedin (originally identified from a sea squirt extract) was approved by the FDA for treatment of soft tissue sarcoma in 2015. This compound was carefully isolated from the natural source and meticulously studied in the laboratory to elucidate the mechanism of anticancer activity. These successes demonstrate the importance of identifying the active compound and understanding the mechanisms of action in order to develop new potential therapies from natural products.

This study has some limitations. Data was based on an observational case series and only includes 10 patients with different risk factors and underlying cirrhosis grade. Additionally, we do not have survival data on these patients. Nevertheless, it demonstrated a signal of tolerance and potential activity in HCC. Furthermore, this study does not include biological correlates such as genetic mutations which would provide potential pathways by which the ME is working. Therefore, future studies with preserved liver function and biospecimen collection for predictive biomarkers exploration are warranted. Finally, alpha-fetoprotein (AFP) is a tumor marker that is commonly elevated in HCC but has limited utility as a prognostic marker. However, the current study provides preliminary evidence of the potential effect of VF-2 on AFP in HCC, suggesting AFP may have value as a marker for clinical response to VF-2 in HCC patients.

In summary, this study indicates that the ME VF-2 may have anticancer activity for some patients with advanced HCC. A significant amount of additional research is needed to further understand this possible effect in order to develop a more effective therapy. Future studies

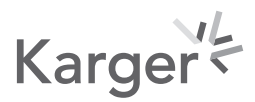


should focus on corroborating this possible anticancer activity, identifying the active compound responsible for the clinical activity seen in HCC and in parallel, identifying predictive markers for response to this treatment.

\section{Acknowledgement}

Helen Moss Foundation and Schoff Family.

\section{Statement of Ethics}

All subjects have given their written informed consent, and the study protocol was approved by the IRB at MD Anderson, Protocol \#DR-0148.

\section{Conflict of Interest Statement}

The authors have no conflicts of interest to declare.

\section{Funding Sources}

None.

\section{Author Contributions}

Richard T. Lee: study design, acquisition of data, data analysis, interpretation of data, manuscript preparation; Peiying Yang: data analysis, interpretation of data, manuscript preparation; Asrar Alahmadi: data analysis, interpretation of data, manuscript preparation; Jennifer McQuade: acquisition of data, data analysis, interpretation of data, manuscript preparation; Eric Yuan: data analysis, interpretation of data, manuscript preparation; Analisa Difeo: data analysis, interpretation of data, manuscript preparation; Goutham Narla: data analysis, interpretation of data, manuscript preparation; Ahmed Kaseb: study design, acquisition of data, data analysis, interpretation of data, manuscript preparation.

\section{References}

1 American Cancer Society.. Global Cancer Facts \& Figures. 4th ed Atlanta: American Cancer Society; 2018.

2 Ryerson AB, Eheman CR, Altekruse SF, Ward JW, Jemal A, Sherman RL, et al. Annual Report to the Nation on the Status of Cancer, 1975-2012, featuring the increasing incidence of liver cancer. Cancer. 2016;122(9): 1312-37.

3 Villanueva A. Hepatocellular Carcinoma. N Engl J Med. 2019;380(15):1450-62.

4 Li DK, Chung RT. Impact of hepatitis C virus eradication on hepatocellular carcinogenesis. Cancer. 2015; 121(17):2874-82.

5 Petrick JL, Kelly SP, Altekruse SF, McGlynn KA, Rosenberg PS. Future of Hepatocellular Carcinoma Incidence in the United States Forecast Through 2030. J Clin Oncol. 2016;34(15):1787-94.

6 Knox JJ, Cleary SP, Dawson LA. Localized and systemic approaches to treating hepatocellular carcinoma. J Clin Oncol. 2015;33(16):1835-44.

7 Kudo M, Finn RS, Qin S, Han KH, Ikeda K, Piscaglia F, et al. Lenvatinib versus sorafenib in first-line treatment of patients with unresectable hepatocellular carcinoma: a randomised phase 3 non-inferiority trial. Lancet. 2018;391(10126):1163-73.

\section{Karger'}


8 Finn RS, Qin S, Ikeda M, Galle PR, Ducreux M, Kim TY, et al. Atezolizumab plus Bevacizumab in Unresectable Hepatocellular Carcinoma. N Engl J Med. 2020;382(20):1894-905.

9 Ebrahim MA, El-Hadaad HA, Alemam OA, Keshta SA. Efficacy and safety of viscum fraxini-2 in advanced hepatocellular carcinoma: a phase II study. Chin-Ger J Clin Oncol. 2010;9(8):452-8.

10 Bergmann L, Aamdal S, Marreaud S, Lacombe D, Herold M, Yamaguchi T, et al. Phase I trial of r viscumin (INN: aviscumine) given subcutaneously in patients with advanced cancer: a study of the European Organisation for Research and Treatment of Cancer (EORTC protocol number 13001). Eur J Cancer. 2008;44(12):1657-62.

11 Bonamin LV, de Carvalho AC, Waisse S. Viscum album (L.) in experimental animal tumors: A meta-analysis. Exp Ther Med. 2017;13(6):2723-40.

12 Yang P, Jiang Y, Pan Y, Ding X, Rhea P, Ding J, et al. Mistletoe extract Fraxini inhibits the proliferation of liver cancer by down-regulating c-Myc expression. Sci Rep. 2019;9(1):6428.

13 Schaub FX, Dhankani V, Berger AC, Trivedi M, Richardson AB, Shaw R, et al. Pan-cancer Alterations of the MYC Oncogene and Its Proximal Network across the Cancer Genome Atlas. Cell Syst. 2018;6(3):282-e2. 